JOURNAL OF COMMUTATIVE ALGEBRA

Volume 2, Number 2, Summer 2010

\title{
ZASSENHAUS RINGS AS IDEALIZATIONS OF MODULES
}

\author{
MANFRED DUGAS
}

\begin{abstract}
A ring $R$ is called a Zassenhaus ring if any homomorphism $\varphi$ of the additive group of $R$ that leaves all left ideals of $R$ invariant, is a left multiplication by some element $a$ of $R$, i.e., $\varphi(x)=a x$ for all $x \in R$. Let $M$ be an $R$ $R$-bimodule. Then the direct sum $R \oplus M$ turns naturally into a ring $R(+) M$ by defining $M M=\{0\}$. This ring is called the idealization of the module $M$, which is an ideal of $R(+) M$. We will investigate conditions under which $R(+) M$ is a Zassenhaus ring.
\end{abstract}

1. Introduction. Let $R$ be a ring and ${ }_{R} M_{R}$ an $R$ - $R$-bimodule.

Then $R(+) M=\left\{\left[\begin{array}{c}r \\ m\end{array}\right]: r \in R, m \in M\right\}$ is a ring with vector addition and multiplication $\left[\begin{array}{c}r \\ m\end{array}\right]\left[\begin{array}{c}r^{\prime} \\ m^{\prime}\end{array}\right]=\left[\begin{array}{c}r r^{\prime} \\ r m^{\prime}+m r^{\prime}\end{array}\right]$, i.e., $R(+) M$ is naturally isomorphic to the ring of matrices $\left\{\left[\begin{array}{cc}r & 0 \\ m & r\end{array}\right]: r \in R, m \in M\right\}$. This ring was first introduced in [11] and is called the idealization of $M$ or a trivial extension of the ring $R$. The very first paper [1] in this journal is an excellent survey article on idealizations, where the ring $R$ is commutative. In this case, any $R$-module $M$ is automatically an $R$ $R$-bimodule. As was pointed out in [1], idealizations provide many nice examples of interesting rings and there is usually some intriguing connection between algebraic properties of $R, M$ and $R(+) M$. We will concern ourselves in this paper with the Zassenhaus property of a ring. Several variations of this theme have been studied in $[2-6]$.

A ring $R$ is called a Zassenhaus ring if any additive endomorphism $\varphi: R \rightarrow R$ such that $\varphi(X) \subseteq X$ for any left ideal $X$ of $R$ is the (left) multiplication by some element of $R$. On the other hand, if $M_{R}$ is a right $R$-module, we define $H(R, M)=\left\{\varphi \in \operatorname{Hom}_{\mathbf{Z}}(R, M): \varphi(r) \in M r\right.$

\footnotetext{
2010 AMS Mathematics subject classification. Primary 13A02, 13A15, Secondary $20 \mathrm{k} 20$.

Keywords and phrases. Idealization of modules, trivial extensions, Zassenhaus rings and modules.

Received by the editors on July 23, 2009, and in revised form on September 23, 2009.

DOI:10.1216/JCA-2010-2-2-139 Copyright (C)2010 Rocky Mountain Mathematics Consortium
} 
for all $r \in R$ \} and call the module $M$ a Zassenhaus module, if each $\varphi \in H(R, M)$ is actually the (left) multiplication by some $\mu \in M$, i.e., $\varphi(r)=\mu r$ for all $r \in R$. (We refer to [8] for some motivation for this nomenclature.)

Here is a partial list of our results:

- If $R(+) M$ is a Zassenhaus ring, then $M_{R}$ is a Zassenhaus module. If $M_{R}$ is also faithful, then $R$ is a Zassenhaus ring.

- $R(+) M$ need not be a Zassenhaus ring, even if $R$ is a Zassenhaus ring and $M$ is a Zassenhaus module.

- There exist Zassenhaus modules $M_{R}$ such that $M_{R}$ is not faithful.

- Let $R$ be a left Ore domain and ${ }_{R} M_{R}$ a bimodule such that ${ }_{R} M$ has rank at least 2 and $M_{R}$ is an $R$-reduced module. Then $R(+) M$ is a Zassenhaus ring if and only if $R$ is a Zassenhaus ring and $M_{R}$ is a Zassenhaus module.

- Let $R$ be an integral domain and $M$ an $R$-reduced $R$-module. Then $R(+) M$ is a Zassenhaus ring if and only if $R$ is a Zassenhaus ring and $M$ is a Zassenhaus module. (Corollary 1 shows that " $R$-reduced" is needed.)

- Assume that the additive group of $R$ is $\mathbf{Z}$-reduced and torsion-free and $M$ contains a strongly pure element. Then $R(+) M$ is a Zassenhaus ring if and only if $M_{R}$ is a Zassenhaus module.

- There are subrings of algebraic number fields that are not Zassenhaus rings and neither are their epimorphic images.

- There are subrings of algebraic number fields that are Zassenhaus rings but not $E$-rings.

- If $R(+) M$ is a Zassenhaus ring, then $R$ need not be a Zassenhaus ring.

\section{Definitions and some general results.}

Definition 1. Let $R$ be a ring, $1 \in R$, and ${ }_{R} M_{R}=M$ an $R-R$ bimodule. We define

$$
\begin{aligned}
\widehat{R} & =\left\{\varphi \in \operatorname{End}_{\mathbf{z}}(R): \varphi(X) \subseteq X \text { for all left ideals } X \text { of } R\right\} \\
& =\left\{\varphi \in \operatorname{End}_{\mathbf{z}}(R): \varphi(r) \in R r \text { for all } r \in R\right\}
\end{aligned}
$$


Note that $R \cdot=\{x \mapsto r x: r \in R\} \subseteq \widehat{R}$. We call $R$ a Zassenhaus ring if $\widehat{R}=R$.

For future reference, we define $\widetilde{R}=\left\{\varphi \in \widehat{R}: \varphi(r) \in R r^{2}\right.$ for all $r \in R\}$.

It is easy to see that $\widetilde{R}$ is a left ideal of $\widehat{R}$ and, if $R$ is commutative, then $\widetilde{R}$ is an ideal of $\widehat{R}$.

Moreover, if $R$ is an integral domain, not a field, then $\widetilde{R} \cap R \cdot=\{0\}$.

In addition, we define

$$
\widehat{M}=\left\{\varphi \in \operatorname{End}_{\mathbf{z}}(M): \varphi(m) \in R m \text { for all } m \in M\right\} .
$$

Finally, let

$$
H(R, M)=\left\{\varphi \in \operatorname{Hom}_{\mathbf{Z}}(R, M): \varphi(r) \in M r \text { for all } r \in R\right\} .
$$

We call $M$ a Zassenhaus module if $H(R, M)=M \cdot=\{x \mapsto m x: m \in$ $M\}$.

Definition 2. A ring $R$ with identity is called a left Ore domain, if $R$ has no zero-divisors, i.e., whenever $r s=0$ for some $r, s \in R$, then $r=0$ or $s=0$, and for any two non-zero elements $u, v \in R$ we have $R u \cap R v \neq\{0\}$.

Let ${ }_{R} M$ be a left module and $m \in M$. We call the element $m$ torsionfree, if $r \in R$ and $r m=0$ implies $r=0$.

We say that ${ }_{R} M$ has rank at least 2 , if ${ }_{R} M$ contains two linearly independent, torsion-free elements. Note that this condition implies that ${ }_{R} M$ be faithful.

Proposition 1. If $M_{R}$ is a faithful Zassenhaus $R$-module, then $R$ is a Zassenhaus ring.

Proof. Let $\alpha \in \widehat{R}$. Let $0 \neq m_{0} \in M$ and define $\beta: R \rightarrow M$ by $\beta(r)=m_{0} \alpha(r)$. Obviously, $\beta \in H(R, M)$. Thus there is some $m \in M$ such that $\beta(r)=m r$ for all $r \in R$. Note that $\alpha(r)=\rho_{r} r$ for some $\rho_{r} \in R$. We infer that $\left(m_{0} \rho_{r}-m\right) r=0$ and, for $r=1$, we have 
$m=m_{0} \rho_{1}$. It follows that $m_{0}\left(\rho_{r}-\rho_{1}\right) r=0$ for all $m_{0} \in M$. Since $M$ is faithful, we have $\alpha(r)-\rho_{1} r=0$ for all $r \in R$. Thus $\alpha=\rho_{1} \cdot \in R$. and $R$ is a Zassenhaus ring.

Remark 1. Let $R$ be a ring and $M_{R}$ an $R$-module such that $M J=\{0\}$ for some ideal $J$ of $R$. Then $M_{R}$ is a Zassenhaus module if and only if $M_{R / J}$ is a Zassenhaus module.

Proof. Assume that $M_{R}$ is a Zassenhaus module. Let $\beta \in$ $H(R / J, M)$. Then there exist $m_{r+J} \in M$ such that $\beta(r+J)=$ $m_{r+J}(r+J)=m_{r+J} r$ for all $r \in R$. Now define $\alpha: R \rightarrow M$ by $\alpha(r)=m_{r+J} r$. It is easy to verify that $\alpha$ is well-defined and $\alpha \in H(R, M)$. This shows that $\alpha(r)=m r$ for a fixed $m \in M$ and all $r \in R$. Thus $\beta(r+J)=\alpha(r)=m r=m(r+J)$, and it follows that the $R / J$-module $M_{R / J}$ is Zassenhaus.

Now assume that $M_{R / J}$ is a Zassenhaus module, and let $\varphi \in$ $H(R, M)$. Then there exist $\mu_{r} \in M$ such that $\varphi(r)=\mu_{r} r$. Note that $\varphi(J)=\{0\}$. Now define $\bar{\varphi}: R / J \rightarrow M$ by $\bar{\varphi}(r+J)=\varphi(r)$. Then $\varphi$ is well defined and $\varphi \in H(R / J, M)=M \cdot$, and there exists some $\mu \in M$ such that $\varphi(r)=\bar{\varphi}(r+J)=\mu(r+J)=\mu r$ for all $r \in R$. This shows that $M_{R}$ is a Zassenhaus module.

Let $M_{R}$ be a Zassenhaus module and $J=\operatorname{ann}_{R}(M)$. Then $M_{R / J}$ is a faithful Zassenhaus module. By Proposition $1, R / J$ is a Zassenhaus ring. Now let $\alpha \in \widehat{R}, \alpha(r)=\rho_{r} r$ for all $r \in R$. Define $\beta: R / J \rightarrow R / J$ by $\beta(r+J)=\rho_{r}(r+J)=\alpha(r)+J$. Note that $\alpha(J) \subseteq J$, which implies that $\beta$ is well defined, and thus $\beta \in \widehat{R / J}$. It follows that there exists some $\rho \in R$ such that $\alpha(r)+J=\beta(r+J)=(\rho+J)(r+J)=\rho r+J$ and thus $(\alpha-\rho \cdot) \in \widehat{R}$. This shows that $(\alpha-\rho \cdot)(R) \subseteq J$. We conclude that $R$ is a Zassenhaus ring provided that $\{\varphi \in \widehat{R}: \varphi(R) \subseteq J\}=\{0\}$.

Definition 3. If $R$ is a ring, then $R^{+}$denotes the additive group of $R$. Then $R^{+}$is Z-reduced, if $\cap_{n \in \mathbf{N}} n R=\{0\}$.

Proposition 2. Let $R$ be a ring such that $R^{+}$is $\mathbf{Z}$-reduced and torsion-free. Then $\widetilde{R}=\{0\}$. 
Proof. Let $\varphi \in \widetilde{R}$. Then there exists an $\rho_{r} \in R$ such that $\varphi(r)=\rho_{r} r^{2}$ for all $r \in R$. Let $n$ be a positive integer. Then $n \rho_{r} r^{2}=n \varphi(r)=$ $\varphi(n r)=\rho_{n r} n^{2} r^{2}$. Thus $n\left(\rho_{r} r^{2}-\rho_{n r} n r^{2}\right)=0$ for all $r \in R$ and all positive integers $n$. This implies $\varphi(r)=\rho_{r} r^{2} \in \cap_{1 \leq n} n R=\{0\}$, since $R^{+}$is $\mathbf{Z}$-reduced.

Proposition 3. Let $R$ be a Zassenhaus ring, $I$ an index set and $M_{R}$ a submodule of the Cartesian product $\Pi=\left(\prod_{I} R\right)_{R}$. Then $M_{R}$ is a Zassenhaus module.

Proof. Let $\beta \in H(R, M)$, and $\beta_{i}$ is the map $\beta$ followed by the projection in the $i$ th coordinate of the cartesian product. Then there exists a $\mu_{r}=\left(\rho_{i}^{(r)}\right)_{i \in I} \in \Pi$ such that $\beta(r)=\mu_{r} r=\left(\rho_{i}^{(r)}\right)_{i \in I} r=$ $\left(\rho_{i}^{(r)} r\right)_{i \in I}$ for all $r \in R$. This implies that $\beta_{i}(r)=\rho_{i}^{(r)} r$ for all $r \in R$ and $\beta \in \widehat{R}$. Thus $\beta_{i}(r)=\rho_{i} r$ for some $\rho_{i} \in R$ and all $r \in R$. This shows that $\beta(r)=\left(\rho_{i}\right)_{i \in I} r$, and since $\beta(1)=\left(\rho_{i}\right)_{i \in I} \in M$ we infer that $\beta \in M \cdot$ and $M$ is a Zassenhaus module.

Remark 2. The above Proposition and the main result in $[\mathbf{7}]$ immediately show the following:

Let $\kappa$ be a cardinal less than the first measurable cardinal and $R$ a Zassenhaus ring with identity such that the additive group of $R$ is slender and $|R|<\kappa$. Then there exist Zassenhaus $R$-modules $G$ of arbitrarily large cardinalities. Moreover, the additive group of $G$ is slender and $\operatorname{End}_{\mathbf{Z}}(G)=R$. This shows that Zassenhaus modules $M_{R}$ exist in abundance if $R$ is a Zassenhaus ring.

Definition 4. Let $R$ be a ring and $M$ an $R$-R-bimodule.

The element $m \in M_{R}$ is pure in $M$ provided that $m \in M u, u \in R$, implies that $u$ is a unit of $R$. The element $m \in M$ is called strongly pure in $M$ if, whenever $s, r$ are non-zero elements of $R$ such that $s m \in M r$, then $s \in R r$. It is easy to see that any strongly pure element is pure. Moreover, if $R_{R}$ is $R$-reduced and $m \in{ }_{R} M_{R}$ is strongly pure, then $m \in_{R} M$ is a torsion-free element.

It can happen that pure implies strongly pure: 
Let $R$ be a commutative ring with identity and view $R$ as a module over itself. If $s \in R$ is a pure element of this module, then $s$ is a unit and thus $s$ is also strongly pure.

There are more examples of modules where pure implies strongly pure:

Let $R$ be a commutative valuation domain, $M$ a torsion-free $R$ module and $m \in M$ a pure element of $M$. Assume $0 \neq s, r \in R$ such that $s m=m^{\prime} r$ for some $m^{\prime} \in M$. If $s \notin R r$, then $r \in R s$ and thus $r=a s$ for some $a \in R$. Then $s m=m^{\prime} a s$ and $m=m^{\prime} a$ for the pure element $m$ implies that $a$ is a unit of $R$ and we get the contradiction $s \in R r$. This shows that the pure element $m \in M$ is strongly pure.

Let $S=R(+) M=\left\{\left[\begin{array}{c}r \\ m\end{array}\right]: r \in R, m \in M\right\}$. We want to compute $\widehat{S}$. To this end, note that $\left[\begin{array}{c}0 \\ M\end{array}\right]$ is an ideal of $S$.

Let $\psi \in \widehat{S}$. Then there exist $\alpha \in \operatorname{End}_{\mathbf{Z}}(R), \beta \in \operatorname{Hom}_{\mathbf{Z}}(R, M)$ and $\gamma \in \operatorname{End}_{\mathbf{Z}}(M)$ such that $\psi$ may be presented as $\psi=\left[\begin{array}{ll}\alpha & 0 \\ \beta & \gamma\end{array}\right]$. Note that $\psi\left(\left[\begin{array}{c}r \\ m\end{array}\right]\right)=\left[\begin{array}{ll}\alpha & 0 \\ \beta & \gamma\end{array}\right]\left[\begin{array}{l}r \\ m\end{array}\right]=\left[\begin{array}{c}\alpha(r) \\ \beta(r)+\gamma(m)\end{array}\right]$. It is easy to see that $\psi \in S \cdot$ if and only if $\psi=\left[\begin{array}{ll}\rho & 0 \\ \mu & \rho\end{array}\right]$ for some $\rho \in R$ and $\mu \in M$.

First we need:

Lemma 1. Let $R$ be a left Ore domain and ${ }_{R} M$ a left $R$-module of rank at least 2 . Then $\widehat{M}=R$, i.e., for any $\varphi \in \widehat{M}$, there is some $\rho \in R$ such that $\varphi(m)=\rho m$ for all $m \in M$.

Proof. Fix a torsion-free element $m \in M$. Then $\varphi(m)=\rho m$ for some $\rho \in R$. Let $m_{1} \in M$ such that $\left\{m, m_{1}\right\}$ is linearly independent over $R$. Then $\varphi\left(m_{1}\right)=\rho_{1} m_{1}$ for some $\rho_{1} \in R$ and there is some $\sigma \in R$ such that $\varphi\left(m+m_{1}\right)=\sigma\left(m+m_{1}\right)$. Since $m, m_{1}$ are $R$ linearly independent we infer that $\rho=\sigma=\rho_{1}$. Now let $\mu \in M$ be another torsion-free element such that $\{m, \mu\}$ is linearly dependent. Then there exist $r, \rho \in R$ such that $r m+\rho \mu=0$ and $r \neq 0 \neq \rho$. We want to show that $\left\{\mu, m_{1}\right\}$ is $R$-linearly independent. To this end, let $r_{0}, r_{1} \in R$ be such that $r_{0} \mu+r_{1} m_{1}=0$. We may assume that 
$r_{0} \neq 0$. Since $R$ is left Ore, we have $R r_{0} \cap R \rho \neq\{0\}$, and there exist $s_{0}, \sigma \in S$ such that $s_{0} r_{0}=\sigma \rho \neq 0$. Note that $s_{0} r_{0} \mu+s_{0} r_{1} m_{1}=0$, and it follows that $\sigma \rho \mu+s_{0} r_{1} m_{1}=0$ and an obvious substitution yields $\sigma(-r m)+s_{0} r_{1} m_{1}=0$ and we conclude $\sigma r=0=s_{0} r_{1}$. Since $R$ is a domain and $\sigma \neq 0 \neq s_{0}$, we have that $r=0=r_{1}$, which shows that $\left\{\mu, m_{1}\right\}$ is $R$-linearly independent and $\varphi\left(m_{1}\right)=\rho m_{1}$. Now the first argument shows that $\varphi(\mu)=\rho \mu$ as well and we have that $\varphi(v)=\rho v$ for all torsion-free elements $v \in M$. Let $\mu \in M$ be a non-torsion-free element, i.e., there is some $0 \neq t \in R$ such that $t \mu=0$. By way of contradiction, we assume that there is some $0 \neq s \in R$ such that $s(m+\mu)=0$. Since $R$ is Ore, there are non-zero elements $x, y \in R$ with $x s=y t \neq 0$. Now $0=x 0=x s m+x s \mu=x s m+y t \mu=x s m$, which contradicts the choice of $m$ being torsion-free. Thus $m+\mu$ is torsion-free and we get $\varphi(\mu)=\varphi((m+\mu)-m)=\rho(m+\mu)-\rho(m)=\rho \mu$. This shows that $\varphi=\rho$.

We are now ready for the following:

Lemma 2. Let $R$ be a left Ore domain, ${ }_{R} M_{R}$ an $R$-R-bimodule such that ${ }_{R} M$ is an $R$-module of rank at least 2 . Let $\alpha \in \operatorname{End}_{\mathbf{z}}(R)$, $\beta \in \operatorname{Hom}_{\mathbf{Z}}(R, M)$ and $\gamma \in \operatorname{End}_{\mathbf{z}}(M)$. Then $\psi=\left[\begin{array}{ll}\alpha & 0 \\ \beta & \gamma\end{array}\right] \in \widehat{R(+) M}$ if and only if

(a) $\alpha \in \widehat{R}$ and there are $\rho_{r} \in R$ such that $\alpha(r)=\rho_{r} r$ for all $0 \neq r \in R$ and

(b) There is some $\rho_{0} \in R$ such that $\gamma(m)=\rho_{0} m$ for all $m \in M$, i.e., $\gamma \in R \cdot$ and

(c) There are $\mu_{r} \in M$ such that $\beta(r)=\mu_{r} r$, i.e., $\beta \in H(R, M)$ and

(d) $\left(\rho_{0}-\rho_{r}\right) m \in M r$ for all $0 \neq r \in R$ and all $m \in M$, i.e., $\left(\rho_{0}-\rho_{r}\right) M \subseteq M r$ for all $0 \neq r \in R$.

Proof. For $r, \rho \in R$ and $m, \mu \in M$ we have $\left[\begin{array}{c}\rho \\ \mu\end{array}\right]\left[\begin{array}{c}r \\ m\end{array}\right]=\left[\begin{array}{cc}\rho & 0 \\ \mu & \rho\end{array}\right]\left[\begin{array}{c}r \\ m\end{array}\right]=$ $\left[\begin{array}{c}\rho r \\ \mu r+\rho m\end{array}\right]$. Now let $\psi=\left[\begin{array}{ll}\alpha & 0 \\ \beta & \gamma\end{array}\right] \in \widehat{R(+) M}$. Then $\psi\left(\begin{array}{c}r \\ m\end{array}\right)=\left[\begin{array}{c}\alpha(r) \\ \beta(r)+\gamma(m)\end{array}\right]=$ $\left[\begin{array}{c}\rho_{r, m} r \\ \mu_{r, m} r+\rho_{r, m} m\end{array}\right]$ for some $\rho_{r, m} \in R$ and $\mu_{r, m} \in M$, and it follows that

$$
\beta(r)+\gamma(m)=\mu_{r, m} r+\rho_{r, m} m \text { for all } r \in R \text { and } m \in M
$$


Moreover, $\alpha(r)=\rho_{r} r$ and $\rho_{r}=\rho_{r, m}$ is independent of $m$ for all $0 \neq r \in R$ and all $m \in M$ since $R$ is a domain, which shows (a).

For $r=0$ we get $\gamma(m)=\rho_{0, m} m$ which shows that $\gamma \in \widehat{M}$ and thus, by Lemma 1 , we have $\gamma(m)=\rho_{0} m$ for all $m \in M$ and $\rho_{0} m=\rho_{0, m} m$ for all $m \in M$. This proves (b).

For $m=0$, we get $\beta(r)=\mu_{r, 0} r$, which shows (c).

We now have $\mu_{r, 0} r+\rho_{0} m=\mu_{r, m} r+\rho_{r} m$ for all $0 \neq r \in R$ and all $m \in M$, i.e.,

$$
\left(\mu_{r, m}-\mu_{r, 0}\right) r=\left(\rho_{0}-\rho_{r}\right) m,
$$

which shows (d).

To show the converse, assume that (a)-(d) hold. Then there exist $\sigma_{r, m}$ such that $\left(\rho_{0}-\rho_{r}\right) m=\sigma_{r, m} r$. Define $\mu_{r, m}=\mu_{r}+\sigma_{r, m}$. The above computations now show that $\psi\left[\begin{array}{c}r \\ m\end{array}\right]=\left[\begin{array}{c}\alpha(r) \\ \beta(r)+\gamma(m)\end{array}\right]=\left[\begin{array}{c}\rho_{r} r \\ \mu_{r} r+\rho_{0} m\end{array}\right]=$ $\left[\begin{array}{cc}\rho_{r} & 0 \\ \mu_{r, m} & \rho_{r}\end{array}\right]\left[\begin{array}{l}r \\ m\end{array}\right]$, since

$$
\begin{aligned}
\mu_{r, m} r+\rho_{r} m & =\left(\mu_{r}+\sigma_{r, m}\right) r+\rho_{r} m=\mu_{r} r+\sigma_{r, m} r+\rho_{r} m \\
& =\mu_{r} r+\left(\rho_{0}-\rho_{r}\right) m+\rho_{r} m=\mu_{r} r+\rho_{0} m .
\end{aligned}
$$

This shows that $\psi \in \widehat{R(+) M}$.

Corollary 1. Let $R=\mathbf{Z}$ and $M=(\mathbf{Q} \oplus \mathbf{Q})_{\mathbf{Z}}$. Then $R$ is a Zassenhaus ring and $M_{R}$ is a Zassenhaus module, but $R(+) M$ is not a Zassenhaus ring.

Proof. Let $\psi=\left[\begin{array}{cc}0 & 0 \\ 0 \operatorname{id}_{M}\end{array}\right]$. Then $\psi \in \widehat{R(+) M}$ by Lemma 2 since $M$ is divisible. It is easy to see that $\psi \notin(R(+) M)$.

The following will come in handy.

Proposition 4. Let $R$ be a ring, $M$ an $R$ - $R$-bimodule and $\beta \in$ $H(R, M)$. Let $\psi=\left[\begin{array}{ll}0 & 0 \\ \beta & 0\end{array}\right]$. The following hold:

(a) $\psi \in \widehat{R(+) M}$ and 
(b) $\psi \in(R(+) M)$. if and only if $\beta \in M \cdot$.

(c) If $R(+) M$ is a Zassenhaus ring, then $M_{R}$ is a Zassenhaus module. If $M_{R}$ is also faithful, then $R$ is a Zassenhaus ring.

Proof. Since $\beta \in H(R, M)$ there exist $\mu_{r} \in M$ such that $\beta(r)=\mu_{r} r$ for all $r \in R$. Thus $\psi\left[\begin{array}{c}r \\ m\end{array}\right]=\left[\begin{array}{c}0 \\ \mu_{r} r\end{array}\right]=\left[\begin{array}{cc}0 & 0 \\ \mu_{r} & 0\end{array}\right]\left[\begin{array}{c}r \\ m\end{array}\right]$ and (a) follows.

We now show (b). If $\beta=\mu$, then we can use the last equation to infer that $\psi \in(R(+) M)$. Assume that $\psi=\left[\begin{array}{ll}\rho & 0 \\ \mu & \rho\end{array}\right] \cdot \in(R(+) M)$. Then $\psi\left[\begin{array}{c}r \\ m\end{array}\right]=\left[\begin{array}{c}\rho r \\ \mu r+\rho m\end{array}\right]=\left[\begin{array}{c}0 \\ \beta(r)\end{array}\right]$. For $r=1$, we get $\rho=0$ and thus $\beta(r)=\mu r$ for all $r \in R$, i.e., $\beta \in M \cdot$

Part (c) is an immediate consequence of parts (a), (b) and Proposition 1.

Definition 3. Let $R$ be a ring and $M_{R}$ an $R$-module. Then $M_{R}$ is called $R$-reduced, if $\cap_{0 \neq r \in R} M r=\{0\}$.

We have:

Proposition 5. Let $R$ be a Zassenhaus Ore domain, $M_{R} R$-reduced and ${ }_{R} M$ of rank at least 2 . Then

$$
\widehat{R(+) M}=\left\{\left[\begin{array}{ll}
\rho \cdot & 0 \\
\beta & \rho \cdot
\end{array}\right]: \rho \in R, \beta \in H(R, M)\right\}=R(+)(H(R, M)) .
$$

Proof. Let $\psi \in \widehat{R(+) M}$. Condition (d) of Lemma 2 now becomes $\left(\mu_{r, m}-\mu_{r, 0}\right) r=\left(\rho_{0}-\rho_{1}\right) m$ for all $m \in M$ and $0 \neq r \in R$ since $R$ is a Zassenhaus ring. Since $M_{R}$ is $R$-reduced and ${ }_{R} M$ is faithful, we infer that $\rho_{0}=\rho_{1}=: \rho$ and $\psi$ has the desired form.

Thus we have:

Proposition 6. Let $R$ be a Zassenhaus left Ore domain, $M_{R}$ reduced and ${ }_{R} M$ of rank at least 2. Then $R(+) M$ is a Zassenhaus ring if and only if $M$ is a Zassenhaus module.

Theorem 1. Let $R$ be a left Ore domain and ${ }_{R} M_{R}$ an $R$ - $R$-bimodule such that ${ }_{R} M$ has rank at least 2 . 
(a) Assume that $M_{R}$ is R-reduced and faithful.

Then $R(+) M$ is a Zassenhaus ring if and only if $R$ is a Zassenhaus ring and $M_{R}$ is a Zassenhaus module.

(b) Let $R^{+}$be $\mathbf{Z}$-reduced and torsion-free. Assume that there is some strongly pure element $m_{0} \in M$.

Then $\widehat{R(+) M}=\left\{\left[\begin{array}{ll}\rho \cdot & 0 \\ \beta & \rho\end{array}\right]: \rho \in R, \beta \in H(R, M)\right\}$.

Thus, $R(+) M$ is a Zassenhaus ring if and only if $M_{R}$ is a Zassenhaus module.

Proof. First we prove (a). If $R(+) M$ is a Zassenhaus ring, then $M_{R}$ and $R$ are Zassenhaus by Proposition 4 (c). To show the converse, assume $\psi=\left[\begin{array}{ll}\alpha & 0 \\ \beta & \gamma\end{array}\right] \in \widehat{R(+) M}$. By Lemma 2, there is some $\rho, \rho_{0} \in$ $R, \mu \in M$ such that $\alpha(r)=\rho r, \beta(r)=\mu r$ for all $r \in R$ and $\gamma(m)=\rho_{0} m$ for all $m \in M$. Moreover, $\left(\rho_{0}-\rho\right) m \in M r$ for all $r \in R, m \in M$. Since ${ }_{R} M$ is faithful and $M_{R}$ is $R$-reduced, we infer $\rho_{0}=\rho$ and thus $\psi=\left[\begin{array}{ll}\rho & 0 \\ \mu & \rho\end{array}\right]$, which shows that $R(+) M$ is a Zassenhaus ring.

We now prove (b). Let $\psi=\left[\begin{array}{ll}\alpha & 0 \\ \beta & \gamma\end{array}\right] \in \widehat{R(+) M}$ with $\alpha(r)=\rho_{r} r$ for all $0 \neq r \in R$ and $\beta(r)=\mu_{r} r$ for some $\mu_{r} \in M$. Moreover, $\gamma(m)=\rho_{0} m$ as in Lemma 2. By Lemma 2 (d), we have that $\left(\rho_{0}-\rho_{r}\right) m_{0} \in M r$, and it follows that $\rho_{0}-\rho_{r} \in R r$ for all $r \in R$ since $m_{0}$ is strongly pure. We infer that $\left(\rho_{0} r-\rho_{r} r\right)=\left(\rho_{0} \cdot-\alpha\right)(r) \in R r^{2}$. Thus $\left(\rho_{0} \cdot\right)-\alpha \in \widetilde{R}=\{0\}$ by Proposition 2. This shows that $\psi=\left[\begin{array}{cc}\rho_{0} & 0 \\ \beta & \rho_{0}\end{array}\right]$ for some $\beta \in H(R, M)$ has the desired form. By Lemma 2, any $\psi$ of this form is in $\widehat{R(+) M}$. We infer that $\widehat{R(+) M}=\left\{\left[\begin{array}{ll}\rho . & 0 \\ \beta & \rho\end{array}\right]: \rho \in R, \beta \in H(R, M)\right\}$. Moreover, $R(+) M$ is a Zassenhaus ring if and only if $M_{R}$ is a Zassenhaus module.

Corollary 1 shows that the hypothesis " $R$-reduced" is needed in the following:

Corollary 2. Let $R$ be an integral domain and $M$ an $R$-reduced $R$ module such that $M$ has rank at least 2. Then $R(+) M$ is a Zassenhaus ring if and only if $R$ is a Zassenhaus ring and $M$ is a Zassenhaus module. 
Note that if $\mathbf{Q} \subsetneq R$ is a field and $M$ is an $R$-vector space, then $H(R, M)=\operatorname{Hom}_{\mathbf{Z}}(M)$. This is an example where neither $R$ nor $M$ is Zassenhaus. We will now show that even Zassenhaus rings may have non-Zassenhaus modules.

Example 1. Let $R$ be a Dedekind domain, not a field, but a Q-algebra. Then there exists an $R$-module $M$ such that $M$ is not Zassenhaus.

Proof. Let $\Pi$ be the set of prime ideals of $R$. For $P \in \Pi$ let $R_{P}$ denote the localization of $R$ at $P$ and $\pi_{P} \in R$ such that $\pi_{P} R_{P}$ is the maximal ideal of the discrete valuation domain $R_{P}$. Note that there are $\mathbf{Q}$ subspaces $C_{P, i}$ of $R_{P}$ such that $\pi_{P}^{n} R_{p}=\oplus_{i \geq n} C_{P, i}$ for all $n=0,1,2, \ldots$. Pick any $\alpha_{P} \in \operatorname{End}_{\mathbf{Q}}\left(R_{P}\right)$ such that $\alpha_{P}\left(C_{P, i}\right) \subseteq C_{P, 2 i}$ for all $i \geq 0$. Note that for any $r \in R_{P}$, there is some $n$ and a unit $u \in R_{P}$ such that $r=\pi_{P}^{n} u$. This implies that $\alpha_{P}(r)=\alpha_{P}\left(\pi_{P}^{n} u\right)=\pi_{P}^{2 n} y$ for some $y \in R_{P}$. Thus $\alpha_{P}(r)=\pi_{P}^{n} y u^{-1}\left(\pi_{P} u\right)=m_{P, r} r$ for $m_{P, r}=\pi_{P}^{n} y u^{-1}$. This shows that $\alpha_{P} \in \widehat{R_{P}}$ but $\alpha_{P} \notin R_{P}$. Now let $M=\prod_{P \in \Pi} R_{P}$, and define $\alpha \in \operatorname{End}_{\mathbf{Q}}(M)$ by $\alpha=\left(\alpha_{P}\right)_{P \in \Pi}$. Let $\widehat{\alpha}$ denote the natural embedding from $R$ into $M$ followed by $\alpha$, i.e., $\widehat{\alpha}(r)=\left(m_{P, r} r\right)=\left(m_{P, r}\right) r=m_{r} r$ for $m_{r}=\left(m_{P, r}\right)_{P \in \Pi}$. Note that $m_{P, r} \in R_{P} r$, and thus there is no $m_{P} \in R_{P}$ such that $m_{P, r}=m_{P}$ for all $r \in R$. This shows that $\widehat{\alpha} \in H(R, M)$, but $\widehat{\alpha} \notin M$. If $R=\mathbf{Q}[x]$ is the rational polynomial ring, then, by [3, Corollary 4], $R$ is a Zassenhaus ring and $M$ is a torsion-free, $R$-reduced $R$-module but not Zassenhaus.

Proposition 7. Let the $\mathbf{Q}$-algebra $R$ be a discrete valuation domain and $M$ an $R$-module. Then $R(+) M$ is not a Zassenhaus ring.

Proof. The case where $R$ is a field follows from [6, Proposition 5]. If $R$ is not a field, we have seen in the proof of Example 1, that there is some $\alpha \in \widehat{R}$ such that $\alpha(r) \in R r^{2}$ for all $r \in R$. It follows from Lemma 2 that $\psi=\left[\begin{array}{ll}\alpha & 0 \\ 0 & 0\end{array}\right] \in \widehat{R(+) M}$ but $\psi \notin(R(+) M)$. 


\section{Subrings of algebraic number fields.}

Notation 1. Let $F=\mathbf{Q}(\omega)$ be an n-dimensional Galois extension of $\mathbf{Q}$ with primitive element $\omega$ and Galois group $G=\left\{g_{1}, g_{2}, \ldots, g_{n}\right\}$ and $\operatorname{id}_{F}=g_{1}$.

Let $\mathfrak{O}_{F}$ denote the ring of algebraic integers of $F$, and let $\left\{a_{1}, a_{2}, \ldots\right.$, $\left.a_{n}\right\}$ be an integral basis of $\mathfrak{O}_{F}$. Let $\Delta=\left[g_{i}\left(a_{j}\right)\right]_{1 \leq i, j \leq n}$.

Note that $\Delta$ is an $n \times n$-matrix with entries in $\mathfrak{O}_{F}$.

Let $p$ be a prime integer such that $p$ does not divide $m_{\Delta}=\operatorname{det}(\Delta)$.

Let $R$ be a full, integrally closed subring of $F$ and $N$ a finite rank torsion-free $R$-module.

For any (prime) ideal $P$ of $\mathfrak{O}_{F}$, let $\operatorname{Fix}(P)=\{g \in G: g(P)=P\}$.

Note that for any $\varphi \in \operatorname{End}_{\mathbf{Q}}(F)$ there are unique $r_{i} \in F$ such that $\varphi=\sum_{1 \leq i \leq n} r_{i} g_{i}$, i.e., $\operatorname{End}_{\mathbf{Q}}(F)=F[G]$, the group ring of $G$ over $F$.

We need the following

Claim 1 [10, Lemma 2.5] (see also [3, Proposition 3]). With the notations as above, let $\varphi=\sum_{1<i<n} r_{i} g_{i} \in \operatorname{End}_{\mathbf{z}}\left(\mathfrak{O}_{F}\right)$, and let $P$ be a prime (maximal) ideal of $\mathfrak{O}_{F}$ lying above the prime integer $p$ (i.e., $p \in P)$ such that $\varphi\left(P^{k}\right) \subseteq P^{k}$ for all positive integers $k$. Then $r_{i}=0$ for all $i$ such that $g_{i} \notin \operatorname{Fix}(P)$.

Claim 2. With the notations as above, let $S=\left(\mathfrak{O}_{F}\right)_{P} \supset \mathfrak{O}_{F}$ be the localization of $\mathfrak{O}_{F}$ at the prime ideal $P$. Then $\widehat{S}=S[\operatorname{Fix}(P)]$, the group ring of $\operatorname{Fix}(P)$ over $S$.

Proof. Since $\left\{P^{k} S: k \geq 1\right\}$ is the list of all non-trivial ideals of the discrete valuation domain $S$, we have that $S[\operatorname{Fix}(P)] \subseteq \widehat{S}$. For the other inclusion, let $\varphi \in \widehat{S}$. There exists a unit $u \in S$ such that $u \varphi\left(\mathfrak{O}_{F}\right) \subseteq \mathfrak{O}_{F}$. Note that $u \varphi\left(P^{k}\right)=u \varphi\left(P^{k} S \cap \mathfrak{O}_{F}\right) \subseteq u \varphi\left(P^{k} S\right) \cap$ $u \varphi\left(\mathfrak{O}_{F}\right) \subseteq P^{k} S \cap \mathfrak{O}_{F}=P^{k}$. This shows that $u \varphi \in \operatorname{End}_{\mathbf{Q}}\left(\mathfrak{O}_{F}\right)$ is such that $u \varphi\left(P^{k}\right) \subseteq P^{k}$ for all $k \geq 1$ and we have $u \varphi \in \mathfrak{O}_{F}[$ Fix $(P)]$ by Claim 1. Thus $\varphi \in u^{-1} \mathfrak{O}_{F}[\operatorname{Fix}(P)] \cap \widehat{S} \subseteq S[\operatorname{Fix}(P)]$. 
Example 2. We will construct a finite rank discrete valuation domain $S$ that is not Zassenhaus and not a Q-algebra. Moreover, $S$ does not admit any Zassenhaus modules $M_{S}$.

Proof. Let $F=\mathbf{Q}(\sqrt{3}, i)=\mathbf{Q}(i+\sqrt{3})=\mathbf{Q}(\sqrt{-1}, \sqrt{-3})$. Let $K=\mathbf{Q}(\sqrt{-1})$ and $L=\mathbf{Q}(\sqrt{-3})$. Then $F=K L$. Note that and $-1 \equiv 3 \bmod 4$ and thus $K$ has discriminant -4 . Moreover, $-3 \equiv 1 \bmod 4$ which implies that the discriminant of $L$ is -3 . This shows that $K, L$ have relatively prime discriminants whose product squared is the discriminant of $F$ and $\mathfrak{O}_{F}=\mathfrak{O}_{K} \mathfrak{O}_{L}$, by $[\mathbf{9}$, page 68, Proposition 17]. Moreover, 5 does not divide the discriminant of $F$, which means that the prime 5 is unramified in $\mathfrak{O}_{F}$. The primitive element $\omega=i+\sqrt{3}$ has minimal polynomial $m(x)=x^{4}-4 x^{2}+16$ and $m(x) \equiv\left(x^{4}+x^{2}+1\right) \bmod 5$. Note that $x^{4}+x^{2}+1=u(x) v(x)$ where $u(x)=x^{2}+x+1$ and $v(x)=x^{2}-x+1$ are irreducible mod5. Let $D=\mathfrak{O}_{F}, P=u(\omega) D+5 D$, and $Q=v(\omega) D+5 D$. Then $P, Q$ are prime ideals of $D$ such that $5 D=P Q$ is the prime factorization of $5 D$.

Note that $u(\omega)=3+\sqrt{3}+i+2 i \sqrt{3}$ and $v(\omega)=3-\sqrt{3}-i-2 i \sqrt{3}$. Let $G=\left\{\operatorname{id}_{F}, \alpha, \beta, \gamma\right\}$ be the Galois group of $F$ where $\alpha(\sqrt{3})=$ $-\sqrt{3}, \alpha(i)=i$ and $\beta(\sqrt{3})=\sqrt{3}, \beta(i)=-i$. Of course, $\gamma=\alpha \beta$. Obviously, $\gamma(u(\omega))=v(\omega)$, which implies $\gamma(P)=Q$. It is easy to verify that $13 \alpha(u(\omega))=13 \alpha(3+\sqrt{3}+i+2 i \sqrt{3})=13(3-\sqrt{3}+i-2 i \sqrt{3})=$ $(3+\sqrt{3}+i+2 i \sqrt{3})(-5+2 \sqrt{3}+12 i-10 i \sqrt{3}) \in P$ and we infer $\alpha(P)=P$ and $\operatorname{Fix}(P)=\left\{\operatorname{id}_{F}, \alpha\right\}$.

Now let $S=D_{P}$ be the localization of $D$ at the prime ideal $P$. Then $S$ is a discrete valuation domain and all non-trivial ideals $J$ of $S$ have the form $J=P^{k} S$ for some $k \geq 1$. Moreover, $\widehat{S}=S[\operatorname{Fix}(P)] \neq S$. Note that none of the rings $S_{n}=S /\left(P^{n} S\right)$ is a Zassenhaus ring. By Proposition 1 and Remark 1, $S$ has no Zassenhaus modules.

Recall that a ring $R$ is an E-ring if $R \cdot=\operatorname{Hom}_{\mathbf{Z}}(R, R)$. Of course, every $E$-ring is a Zassenhaus ring. The results in this section and in [10] allow us to find many examples of Zassenhaus rings that are not $E$-rings. We still use Notation 1. It is well known that $S=\mathfrak{O}_{F}$ is not an $E$-ring but a Zassenhaus ring. Let $\Pi$ be a (finite) set of prime ideals of $S$ such that $\sigma \in G$ and $\sigma(P)=P$ for all $P \in \Pi$ implies that $\sigma=\operatorname{id}_{F}$. Then the localization $R=S_{\Pi}$ is a Zassenhaus ring. It can easily be arranged that $\rho(\Pi)=\Pi$ for some $\operatorname{id}_{F} \neq \rho \in G$. In this case, $R$ is not an $E$-ring. 
The module $N$ over an ( $E$-ring) $R$ is called an $E$-module, if $\operatorname{Hom}_{\mathbf{Z}}(R, N)=N \cdot$. Trivially, any $E$-module is a Zassenhaus module. $E$-modules of finite rank were studied in $[\mathbf{1 0}]$. It is easy to check that the results in $[\mathbf{1 0}$, Section 2] all hold if one replaces " $E$-module" by "Zassenhaus module" and " $\operatorname{Hom}_{\mathbf{Z}}(R, N)$ " by " $H(R, N)$ ". The same can be said about the results in $[\mathbf{1 0}$, Section 3]. We illustrate this with the following:

Example 3. Let $F$ be a quadratic number field and $p$ a prime integer such that $p \mathfrak{O}_{F}=P Q$ for two distinguished prime ideals of $\mathfrak{O}_{F}$. Let $G=\left\{\operatorname{id}_{F}, \sigma\right\}$ be the Galois group of $F$. Then $\sigma(P)=Q$ and it follows that $S=\left(\mathfrak{O}_{F}\right)_{\{P, Q\}}$ is a Zassenhaus ring but not an $E$-ring. The ring $S$ is a subring of the ring $R=\left(\mathfrak{O}_{F}\right)_{P}$ and thus $R$ is an $S$-module. We will show that $R_{S}$ is a Zassenhaus module. It is enough to show that $\sigma \notin H(S, R)$. By way of contradiction, assume otherwise and pick $0 \neq x \in P-\sigma^{-1}(P \cap Q)$. Then $\sigma(x)=\left[\sigma(x) x^{-1}\right] x \in R x$, which implies that $\sigma(x) x^{-1} \in R$ and $\sigma(x) \in Q-P$ is a unit in $R$. Thus $x^{-1} \in R$ and we get the contradiction $1=x^{-1} x \in P$. Of course, this example can be vastly generalized.

4. The case of $S=\mathbf{Z}[x]$. In this section, $S$ will always denote the integer polynomial ring $S=\mathbf{Z}[x]$. We define $J=\{(f(x) / g(x))$ : $f(x), g(x) \in S, g(x)$ primitive $\}$. Recall that $S$ is a subring of the integral domain $J$, and all ideals $I$ of $J$ have the form $I=n J$ for some integer $n$.

Here is another Zassenhaus ring which admits a non-Zassenhaus module:

Example 4. There exists a commutative ring $R$ such that $R$ is not a Zassenhaus ring, but some epimorphic image of $R$ is a Zassenhaus ring.

Proof. Note that $J$ is a ring and every element of $J$ is of the form of an integer times a unit of $J$. Define $\varphi \in \operatorname{Hom}_{\mathbf{Z}}(S, J)$ by $\varphi(f(x))=f\left(x^{2}\right)$. Let $y=n g(x) \in S$ with $g(x)$ a primitive polynomial. Then $g(x)$ is a unit in $J$ and we have $\varphi(y)=n g\left(x^{2}\right) g(x)^{-1} g(x)=\left(g\left(x^{2}\right) / g(x)\right) y$ where 
$\left(g\left(x^{2}\right) / g(x)\right) \in J$ and it follows that $\varphi \in H(S, J)-(J \cdot)$. Now consider $R=S(+) J$. By Proposition 4 (c), the ring $R$ is not a Zassenhaus ring, but $S \cong R / J$ is a Zassenhaus ring.

Let $S \subset J$ be as above, and let ${ }_{S} M_{J}$ be an $S$ - $J$-bimodule. We will show that $H\left(S, M_{S}\right)=\operatorname{Hom}_{\mathbf{Z}}(S, M)$ :

Assume that $\varphi \in \operatorname{Hom}_{\mathbf{Z}}(S, M)$. Let $y=n g \in S$ be such that $n \in \mathbf{N}$ and $g \in S$ is primitive. Then $\varphi(y)=\varphi(g) g^{-1} n g=\left(\varphi(g) g^{-1}\right) y$ and $\varphi(g) g^{-1} \in M$ since $g^{-1} \in J$. This shows that $\varphi \in H(S, M)$.

If $R$ is a ring with identity, then $R$ is naturally a subring of $\widehat{R}$. This allows us to use transfinite induction to define an ascending chain of rings $\left\{R^{(\alpha)}: \alpha\right.$ an ordinal $\}$ as follows: Let $R^{(0)}=R$ and $R^{(\alpha+1)}=\widehat{R^{(\alpha)}}$. For limit ordinals $\lambda$, we define $R^{(\lambda)}=\cup_{\alpha<\lambda} R^{(\alpha)}$. There is an example in $[3]$ for which this transfinite chain never terminates, i.e., $R^{(\alpha)} \varsubsetneqq R^{(\alpha+1)}$ for all ordinals $\alpha$. We will present another such example, where all the rings in the transfinite chain are idealizations of $S=\mathbf{Z}[x]$-modules.

Recall that by Proposition 5, we have

$$
\widehat{S(+) M}=\left\{\left[\begin{array}{cc}
\rho \cdot & 0 \\
\beta & \rho \cdot
\end{array}\right]: \rho \in S, \beta \in H\left(S, M_{S}\right)\right\}=S(+)\left(\operatorname{Hom}_{\mathbf{Z}}(S, M)\right) .
$$

For $s \in S, \varphi \in \operatorname{Hom}_{\mathbf{Z}}(S, M), j \in J$, define $(s \varphi j)(x)=s \varphi(x) j$ for all $x \in S$. Then $\varphi \in \operatorname{Hom}_{\mathbf{Z}}(S, M)$ and $\operatorname{Hom}_{\mathbf{Z}}(S, M)$ becomes an $S$-J-bimodule. We may define $R^{(0)}=S(+) J$ and $R^{(1)}=\widehat{R^{(0)}}=$ $S(+)\left(\operatorname{Hom}_{\mathbf{Z}}(S, J)\right)$. Note that $J$ naturally embeds into $\operatorname{Hom}_{\mathbf{Z}}(S, J)$ via $j(s)=s j$ for all $s \in S$. This induces a natural embedding of $R^{(0)}$ into $R^{(1)}$. More generally, given ${ }_{S} M_{J}$ there is a natural embedding of $M$ into $\operatorname{Hom}_{\mathbf{Z}}(S, M)$ by $m(s)=m s$ for all $m \in M, s \in S$. This allows us to define $R^{(\alpha+1)}=S\left(\widehat{+\left(M^{(\alpha)}\right.}=S(+) \operatorname{Hom}_{\mathbf{Z}}\left(S, M^{(\alpha)}\right)=S(+) M^{(\alpha+1)}\right.$ with $M^{(0)}=J$. Note that $M^{(\alpha)} \varsubsetneqq M^{(\alpha+1)}$ via the natural embedding. Note that the chain $\left\{R^{(\alpha)}: \alpha\right.$ an ordinal $\}$ never terminates.

On the other hand we have the somewhat surprising:

Lemma 3. Let $A$ be a torsion-free, Z-reduced abelian group. Then $M_{S}=A \otimes \mathbf{z} S$ is a Zassenhaus module. 
Proof. Let $s=\sum_{0<i<N} k_{i} x^{i} \in S$ be such that $k_{0} \neq 0$. Let $\varphi \in H(S, M)$. Then there are $a_{n, \alpha} \in A$ such that

$$
\varphi\left(x^{n}\right)=\sum_{0 \leq \alpha \leq d_{n}} a_{n, \alpha} \otimes x^{\alpha} \in M=\oplus_{\alpha \geq 0}\left(A \otimes x^{\alpha}\right) .
$$

Since $\varphi \in H(S, M)$, there is a $c_{s} \in M$ such that $\varphi(s)=c_{s} s$ for all $s \in S$. Let $c_{s}=\sum_{0 \leq \beta \leq N_{s}} \ell_{s, \beta} \otimes x^{\beta}$.

We compute

$$
\begin{aligned}
\varphi(s) & =\sum_{i} k_{i} \varphi\left(x^{i}\right)=\sum_{i} k_{i}\left(\sum_{\alpha} a_{i, \alpha} \otimes x^{\alpha}\right) \\
& =\sum_{\alpha}\left(\left(\sum_{i} k_{i} a_{i, \alpha}\right) \otimes x^{\alpha}\right) .
\end{aligned}
$$

On the other hand,

$$
\begin{aligned}
\varphi(s) & =c_{s} s=\left(\sum_{0 \leq \beta \leq N_{s}} \ell_{s, \beta} \otimes x^{\beta}\right)\left(\sum_{0 \leq i \leq N} k_{i} x^{i}\right) \\
& =\sum_{i, \alpha} \ell_{s, \beta} k_{i} \otimes x^{i+\beta} \\
& =\sum_{\alpha}\left(\left(\sum_{i} \ell_{s, \alpha-i} k_{i}\right) \otimes x^{\alpha}\right) .
\end{aligned}
$$

Thus, for all $\alpha \geq 0$, we have

(*) $\sum_{0 \leq i \leq \alpha} \ell_{s, \alpha-i} k_{i}=\sum_{i \geq 0} k_{i} a_{i, \alpha}=\sum_{0 \leq i \leq N} k_{i} a_{i, \alpha}$.

Note that $\varphi\left(k_{0}\right)=\sum_{\alpha} \ell_{1, \alpha} k_{0} \otimes x^{\alpha}=\sum_{\alpha} k_{0} a_{0, \alpha} \otimes x^{\alpha}$ and it follows that $a_{0, \alpha}=\ell_{1, \alpha}$ for all $\alpha$.

Now let $t(\alpha, s)=-\sum_{1 \leq i \leq \alpha} k_{i} a_{0, \alpha-i}+\sum_{0 \leq i \leq N} k_{i} a_{i, \alpha} \in A$. Since $A$ is Z-reduced, there is some natural number $\|t(s, \alpha)\|$ such that $t(\alpha, s) \notin\|t(\alpha, s)\| A$ provided that $t(\alpha, s) \neq 0$.

Let $w_{s}=\operatorname{lcm}\left\{\|t(\alpha, s)\|: t(\alpha, s) \neq 0,1 \leq \alpha \leq N_{s}\right\}$.

$(* *)$ Assume that $w_{s}$ divides the integer $k_{0}=s(0)$.

We will show that 
$(* * *) \ell_{s, \alpha}=a_{0, \alpha}$ for all $\alpha \geq 0$.

We proceed by induction over $\alpha$. For $\alpha=0$ we have the equation $\ell_{s, 0} k_{0}=k_{0} a_{0,0}+\sum_{i>1} k_{i} a_{i, 0}$, and it follows that $k_{0}^{-1}\left(\sum_{i>1} k_{i} a_{i, 0}\right) \in A$ no matter how the $k_{i}$ 's are chosen. Since $A$ is Z-reduced, we infer that $a_{i, 0}=0$ for all $i \geq 1$, and we have that $\ell_{s, 0}=a_{0,0}$ for all $s \in S$. This shows that $(* * *)$ holds for $\alpha=0$.

Now assume that $(* * *)$ holds for all $0 \leq \beta<\alpha$. Now $(*)$ becomes $\ell_{s, \alpha} k_{0}=k_{0} a_{0, \alpha}-\sum_{1 \leq i \leq \alpha} k_{i} a_{0, \alpha-i}+\sum_{0 \leq i \leq N} k_{i} a_{i, \alpha}$, and thus $k_{0}\left(\ell_{s, \alpha}-a_{0, \alpha}\right)=t(\alpha, s)$. If $\ell_{s, \alpha}-a_{0, \alpha} \neq 0$, we get the contradiction $k_{0}^{-1} t(\alpha, s) \in A$ by $(* *)$. This shows that $\ell_{s, \alpha}=a_{0, \alpha}$ for all $s \in S$ that satisfy $(* *)$, i.e., $s(0)$ is "big enough."

For such an element $s \in S$ we have that $c_{s}=\sum_{\alpha} \ell_{s, \alpha} \otimes x^{\alpha}=$ $\sum_{\alpha} a_{0, \alpha} \otimes x^{\alpha}=\varphi(1)=\varphi\left(x^{0}\right)$. Now let $v \in S$. Then there exists some $k \in \mathbf{Z}$ such that $k+v$ satisfies $(* *)$. As we just have seen, this implies $\varphi(1) k+\varphi(v)=\varphi(k+v)=\varphi(1)(k+v)=\varphi(1) k+\varphi(1) v$ and the desired equation $\varphi(v)=\varphi(1) v$ follows for all $v \in S$. Thus $M_{S}$ is a Zassenhaus module.

We also need:

Lemma 4. Let $S=\mathbf{Z}[x] \subseteq R \subseteq V$ be rings with torsion-free additive groups and ${ }_{R} M_{S}=V \otimes \mathbf{z} S$. Let $0 \neq t \in R$ and $s \in S$ be such that $t \otimes 1=m s$ for some $m \in M$. Then $s \in \mathbf{Z}, m=u \otimes 1$ for some $u \in V$ and $t=u s$.

Proof. Let $s=\sum_{0 \leq i \leq N} k_{i} x^{i}$. There exist finitely many $v_{j} \in V$ such that $m=\sum_{j} v_{j} \otimes x^{j}$. Then $t \otimes 1=m s=\left(\sum_{j} v_{j} \otimes x^{j}\right)\left(\sum_{0 \leq i \leq N} k_{i} x^{i}\right)=$ $\sum_{\alpha}\left(\sum_{0 \leq i \leq \alpha} v_{\alpha-i} k_{i}\right) \otimes x^{\alpha}$. This implies that $t=v_{0} k_{0}$ and $k_{0} \neq 0$ since $t \neq 0$. We have

(*) $0=\sum_{0<i<\alpha} v_{\alpha-i} k_{i}$ for all $\alpha \geq 1$. An easy induction shows that $v_{j}=v_{0} q_{j}$ for some $q_{j} \in \mathbf{Q}$ with $q_{0}=1$. Now $t=v_{0}\left(q_{0} k_{0}\right)$ and $v_{0}\left(\sum_{0 \leq i \leq \alpha} q_{\alpha-i} k_{i}\right)=0$. Let $g(x)=\sum_{j} q_{j} x^{j} \in \mathbf{Q}[x]$. The equations $(*)$ imply that $g(x) s=q_{0} k_{0}=k_{0}$. We infer that $g(x)=1$ and $s=k_{0}$ are constant polynomials. It follows that $m=v_{0} \otimes 1, s=k_{0}$ and $t=v_{0} k_{0}$ as claimed. 
We also need

Lemma 5. Let $M=(R \otimes \mathbf{z} S) e_{1} \oplus(R \otimes \mathbf{z} S) e_{2}$ and $\widehat{M}=\{\varphi \in$ $\operatorname{Hom}_{\mathbf{Z}}(M, M): \varphi(m) \in R m$ for all $\left.m \in M\right\}$. If $\varphi \in \widehat{M}$, then there exists some $\rho \in R$ such that $\varphi(m)=\rho m$ for all $m \in M$.

Proof. Let $\varphi \in \widehat{M}$. Then there exist $\rho_{s, i} \in R$ such that $\varphi((1 \otimes$ $\left.s) e_{i}\right)=\left(\rho_{s, i} \otimes s\right) e_{i}$ for $i=1,2$ and $\varphi\left((1 \otimes s) e_{1}+(1 \otimes s) e_{2}\right)=$ $\rho_{s}\left((1 \otimes s) e_{1}+(1 \otimes s) e_{2}\right)$, and it follows that $\rho_{s, 1}=\rho_{s}=\rho_{s, 2}$ for all $s \in S$. Now $\varphi\left((1 \otimes s) e_{1}+(1 \otimes t) e_{2}\right)=\rho_{s, t}\left((1 \otimes s) e_{1}+(1 \otimes t) e_{2}\right)=$ $\rho_{s}(1 \otimes s) e_{1}+\rho_{t}(1 \otimes t) e_{2}$, and it follows that $\rho_{s}=\rho_{t}$ for all $s, t \in R$. Thus there is an element $\rho \in R$ such that $\varphi(1 \otimes s)=\rho(1 \otimes s)=\rho \otimes s$ for all $s \in S$. Let $r \in R$, and compute $\varphi\left((1 \otimes s) e_{1}+(r \otimes s) e_{2}\right)=$ $\tau_{r, s}\left((1 \otimes s) e_{1}+(r \otimes s) e_{2}=(\rho \otimes s) e_{1}+t_{r, s}(r \otimes s) e_{2}\right.$ where $\varphi\left((r \otimes s) e_{2}=\right.$ $t_{r, s}(r \otimes s) e_{2}$. It follows that $\rho=\tau_{r, s}$ and $\rho r=t_{r, s} r$. Therefore, $\varphi\left((r \otimes s) e_{2}=\left(t_{r, s} r \otimes s\right) e_{2}=(\rho r \otimes s) e_{2}=\rho\left((r \otimes s) e_{2}\right)\right.$. In a similar fashion, one can show that $\varphi\left((r \otimes s) e_{1}=\rho\left((r \otimes s) e_{1}\right)\right.$ for all $r \in R, s \in S$ and $R \otimes \mathbf{z} S$ is additively generated by elements of this form. This shows that $\varphi(m)=\rho m$ for all $m \in M$.

Now we are ready to prove:

Theorem 2. There exists a commutative ring $R$ and $R$-module $M$ of rank at least 2 , such that $R$ is not a Zassenhaus ring, but $R(+) M$ is a Zassenhaus ring.

Proof. Let $S=\mathbf{Z}[x]$, and let $J$ be as defined at the beginning of this section. By Example 4, the ring $R=S(+) J$ is not a Zassenhaus ring.

Let ${ }_{R} M_{S}=(R \otimes \mathbf{z} S) e_{1} \oplus(R \otimes \mathbf{z} S) e_{2}$, which is naturally a $R$ - $S$ bimodule, which turns into an $R$ - $R$-bimodule ${ }_{R} M_{R}$ by setting $M J=$ $\{0\}$, i.e., $M_{R}$ is not faithful but ${ }_{R} M$ has rank at least 2 .

Define $T=R(+) M$.

Recalling the notations of Lemma 2, let $\psi=\left[\begin{array}{ll}\alpha & 0 \\ \beta & \gamma\end{array}\right] \in \widehat{T}$ and $m_{0}=(1 \otimes 1) e_{1} \in M$. Then $\psi\left[\begin{array}{c}r \\ m\end{array}\right]=\left[\begin{array}{cc}\rho_{r, m} & 0 \\ \mu_{r, m} & \rho_{r, m}\end{array}\right]\left[\begin{array}{c}r \\ m\end{array}\right]=\left[\begin{array}{c}\rho_{r, m} r \\ \mu_{r, m} r+\rho_{r, m} m\end{array}\right]$, 
and it follows that $\alpha(r)=\rho_{r, m} r$ and $\beta(r)+\gamma(m)=\mu_{r, m} r+\rho_{r, m} m$ for all $r \in R, m \in M$.

For $r=0$, we get $\gamma(m)=\mu_{0, m} m$, which means that $\gamma \in \widehat{M}$ and by Lemma 5 , there is some $\rho_{0} \in R$ such that $\gamma(m)=\rho_{0} m=\mu_{0, m} m$ for all $m \in M$.

For $m=0$, we get $\beta(r)=\mu_{r, 0} r$ for all $r \in R$ and thus $\beta \in H(R, M)$. By Lemma 3 and Remark $1, M_{R}$ is a Zassenhaus module and thus there is some $\mu_{0} \in M$ such that $\beta(r)=\mu_{0} r=\mu_{r, 0} r$ for all $r \in R$.

Now we have $\mu_{0} r+\rho_{0} m=\mu_{r, m} r+\rho_{r, m} m$.

It follows $\left(\rho_{0}-\rho_{r, m}\right) m=\left(\mu_{r, m}-\mu_{0}\right) r$ for all $r \in R, m \in M$. We choose $m=m_{0}$ and obtain $\left(\rho_{0}-\rho_{r, m_{0}}\right)(1 \otimes 1)=b r$ for some $b \in R \otimes \mathbf{z} S$. Now apply Lemma 4 and infer that $\rho_{0}=\rho_{r, m_{0}}$ for all $r \in R-(\mathbf{Z} \oplus J)$. This shows that $\rho_{0}=\rho_{r, m_{0}}$ and $\alpha(r)=\rho_{0} r$ for all $r=s+j \in R$ such that $s \in S$ is not constant. Let $z \in \mathbf{Z}, j \in J$ and $\sigma \in S$ any polynomial of positive degree.

Then $\alpha(z+j)=\alpha((z-\sigma+j)+\sigma)=\alpha(z-\sigma+j)+\alpha(\sigma)=$ $\rho_{0}(z-\sigma+j)+\rho_{0} \sigma=\rho_{0}(z+j)$. This shows that $\alpha=\rho_{0} \cdot \in R$.

It follows that $\psi=\left[\begin{array}{cc}\rho_{0} & 0 \\ \mu & \rho_{0}\end{array}\right] \in T \cdot$, and we have that $T$ is a Zassenhaus ring.

\section{REFERENCES}

1. D.D. Anderson and M. Winders, Idealization of a module, J. Comm. Algebra. 1 (2009), 3-56.

2. J. Buckner and M. Dugas, Quasi-localizations of Z, Israel J. Math. 160 (2007), 349-370.

3. - Left rigid rings, J. Algebra 309 (2007), 192-206.

4. - Rings with Zassenhaus families of ideals, Comm. Algebra 36 (2008), $2133-2142$.

5. - Group algebras with Zassenhaus families of right ideals, Houston J. Math., to appear.

6. ——, Zassenhaus algebras, Rocky Mountain J. Math., to appear.

7. M. Dugas, Large E-modules exist, J. Algebra 142 (1991), 405-413.

8. M. Dugas and R. Göbel, An extension of Zassenhaus' theorem on endomorphism rings, Fund. Math. 194 (2007), 239-251.

9. S. Lang, Algebraic number theory, Second edition, Grad. Texts Math., SpringerVerlag, New York, 1994. 
10. A. Mader and C. Vinsonhaler, Torsion-free E-modules, J. Algebra 115 (1988), 401-411.

11. M. Nagata, Local rings, Interscience Tracts Pure Appl. Math. 13, John Wiley and Sons, New York, 1962.

Department of Mathematics, Baylor University, Waco, TX 76798

Email address: Manfred_Dugas@baylor.edu 